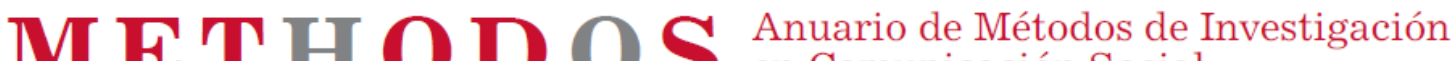 en Comunicación Social
}

Mayo 2020

\section{Entrevistas semiestructuradas con NVivo: pasos para un análisis cualitativo eficaz}

\author{
Carlos Lopezosa \\ Universitat Pompeu Fabra \\ carlos.lopezosa@upf.edu \\ ORCID: 0000-0001-8619-2194
}

DOI: 10.31009/methodos.2020.i01.08

Lopezosa, C. (2020). Entrevistas semiestructuradas con NVivo: pasos para un análisis cualitativo eficaz. En: Lopezosa, C.; Díaz-Noci, J.; Codina, L. (ed.). Anuario de Métodos de Investigación en Comunicación Social, n.1 (p.88-97). Barcelona: DigiDoc-Universitat Pompeu Fabra

Esta obra está bajo una Licencia

Creative Commons Atribución

4.0 Internacional.

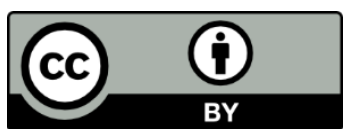





\title{
Entrevistas semiestructuradas con NVivo: pasos para un análisis cualitativo eficaz
}

\author{
Carlos Lopezosa \\ Universitat Pompeu Fabra \\ carlos.lopezosa@upf.edu \\ ORCID: 0000-0001-8619-2194
}

\section{RESUMEN}

Este informe sostiene que el software de análisis de datos cualitativos NVivo puede ser una herramienta muy útil para analizar e interpretar entrevistas semiestructuradas. Para ello, se plantea un ejemplo práctico de cómo aplicar esta herramienta a investigaciones cualitativas, utilizando como caso 10 entrevista sobre posicionamiento en buscadores en cibermedios a un experto en este campo. Asimismo, en este trabajo se explica qué procesos hay que tener en cuenta para manejar e interpretar adecuadamente los datos que proporcionen la codificación de las entrevistas con NVivo. El proceso incluye estrategias de interpretación de datos en nodos de árbol, y en nubes de categorías. El artículo concluye con una serie de recomendaciones para que los investigadores sean capaces de sacar el mayor provecho posible a este software.

\section{PALABRAS CLAVE}

Entrevistas semiestructuradas, NVivo, metodología cualitativa, software de apoyo a la investigación

\section{Semi-estructured interview with Nvivo: Steps for an efficient qualitative analysis}

\section{ABSTRACT}

This report argues that NVivo qualitative data analysis software can be a very useful tool for analyzing and interpreting semi-structured interviews. For this purpose, a practical example of how to apply this tool to qualitative research is presented, using an expert in this field as case study 10 interviews on search engine positioning in cybermedias. We establish the processes which must be taken into account in order to handle properly and interpret the data that provide the coding of interviews with Nvivo. The process includes data interpretation strategies in tree nodes and category clouds. The article concludes with a series of recommendations for researchers to make the most of this software.
Entrevistes semi-estructurades amb NVivo: Passos per a un anàlisis eficaç

\section{KEYWORDS}

Semi-structured interviews, NVivo, qualitative methodology, research support software.

\section{RESUM}

Aquest informe sosté que el programari d'anàlisi de dades qualitatives NVivo pot ser una eina molt útil per analitzar i interpretar entrevistes semiestructurades. Per a això, es planteja un exemple pràctic de com aplicar aquesta eina a investigacions qualitatives, utilitzant com cas 10 entrevista sobre posicionament en cercadors en cibermitjans a un expert en aquest camp. Així mateix, en aquest treball s'explica quins processos cal tenir en compte per gestionar i interpretar adequadament les dades que proporcionin la codificació de les entrevistes amb NVivo. El procés inclou estratègies d'interpretació de dades en nodes d'arbre, i en núvols de categories. L'article conclou amb una sèrie de recomanacions perquè els investigadors siguin capaços de treure el major profit possible a aquest programari.

\section{PARAULES CLAU}

Entrevistes semiestructurades, NVivo, metodologia qualitativa, programari de suport a la investigació.

DOI: $10.31009 /$ methodos.2020.i01.08 


\section{Las entrevistas semies- tructuradas en el contexto de la investigación}

La entrevista es un instrumento de gran eficacia para desarrollar investigaciones cualitativas y tiene como función principal recabar datos que después podremos aplicar a nuestros estudios. Se trata de una técnica que se caracteriza por tratarse de una conversación más o menos dirigida (dependiente del tipo de entrevista) entre el investigador (emisor) y el sujeto de estudio (receptor) con un fin siempre bien determinado y enfocado a la resolución de los objetivos y preguntas de investigación de trabajos. Para alcanzar este fin el investigador plantea interrogantes al receptor para que éste le dé su opinión, los responda o los resuelva, según el caso.

Existen tres tipos de entrevistas que se pueden aplicar a lo largo de una investigación, cada una de ellas cuenta con sus propias ventajas e inconvenientes, por lo que optar por una u otra condicionará en mayor o menos medida el resultado de nuestro estudio (Díaz-Bravo et al. 2013). A continuación, explicamos brevemente cada una las entrevistas que podemos realizar:

- Entrevistas estructuradas: Se trata de una entrevista que cuenta con preguntas fijadas de antemano con un orden específico y con posibles respuestas cerradas. Los entrevistados deben seleccionar del listado de respuestas las que consideren más adecuadas. Es un tipo de entrevista muy rígida, destaca por permitir una gran sistematización en los resultados y por tanto un alto grado de objetividad, sin embargo, en algunas ocasiones la falta de interpretación por dicha rigidez, ofrece resultados menos ricos que otro tipo de entrevistas.

- Entrevistas semiestructuradas: Tiene menor rigidez que las entrevistas estructuradas, ya que cuentan con preguntas fijas, pero en este caso los entrevistados pueden contestar libremente sin necesidad de elegir una respuesta específica como sucede en las entrevistas estructurada. Incluso los investigadores pueden interactuar y adaptarse a los entrevistados y a sus respuestas, en definitiva, son entrevistas más dinámicas, flexibles y abiertas, y por tanto permiten una mayor interpretación de los datos que con las entrevistas estructuradas.

- Entrevistas no estructuradas: Son las entrevistas más flexibles ya que las preguntas son más abiertas. Los entrevistados responden sin estar dirigidos, lo que ayuda a que vayan más allá de las propias preguntas, esto incluye desviarse en las contestaciones, divagar, etc. Es un tipo de entrevista de gran riqueza a nivel interpretativo, sin embargo, en ocasiones parte de las respuestas pueden desvincularse totalmente del objeto de estudio, y por tanto el investigador tiene la misión de eliminar el contenido de poco o nulo valor y rescatar el que sí lo tiene. Muchas ocasiones esto se puede volver tedioso y/o complicado.

Es importante destacar que no hay que confundir las entrevistas con los cuestionarios, ya que, aunque cuentan con algunas similitudes, las entrevistas permiten, en mayor o menor grado, interactuar con el receptor, ya sea aclarando dudas, o interviniendo directamente en la conversación para dirigir o enfatizar en algún punto o tema propuesto, mientras que las encuestas no. Todo esto hace que la entrevista tenga un grado de eficacia mayor que el cuestionario.

A su vez, y una vez visto las diferencias entre las encuestas y las entrevistas, y los tipos de entrevistas, cabe destacar que, actualmente, la entrevista semiestructurada está considerada la que mayor interés suscita dentro del mundo académico. Esto se debe principalmente a su grado de flexibilidad a la hora de resolver las preguntas, ya que las respuestas que se dan no son ni demasiado rígida ni demasiado abierta. Esto se traduce en una mayor participación de los entrevistados en ofrecer su punto de vista, una mayor uniformidad de respuestas, y por tanto mayor facilidad para que los investigadores puedan interpretar las respuestas en el contexto de su estudio.

En definitiva, la entrevista es una metodología cualitativa que nos ayuda a recabar datos muy detallados y profundos principalmente porque se adaptan al entrevistado desde aspectos muy variados como , su contexto, su conocimiento sobre el tema, sus creencias, etc.

Ahora bien, ¿cómo podemos identificar qué datos son los más relevantes cuando realizamos una investigación en base a entrevistas a expertos? ¿Cómo saber si las interpretaciones realizadas en las entrevistas semiestructuradas y la elección de los datos son lo que realmente aportan valor a nuestras investigaciones?

La respuesta está en la investigación exhaustiva del contenido de las respuestas de los entrevistados. Se trata de analizar punto por punto cada una de las respuestas de los entrevistados y resaltar los puntos de convergencia y divergencia que se dan entre todos ellos, sistematizarlos y categorizanrlos hasta reducirlos a conclusiones solventes, específicas, y adecuadas que resuelvan nuestros objetivos y preguntas de investigación.

Si bien es cierto que esta labor se viene realizando en base a una lectura (en caso de entrevistas transcritas), o una escucha (en caso de entrevistas en audio) concienzuda, cada vez es más frecuente encontrar programas informáticos que ayudan a categorizar y automatizar los resultados de las entrevistas. Con ello, 


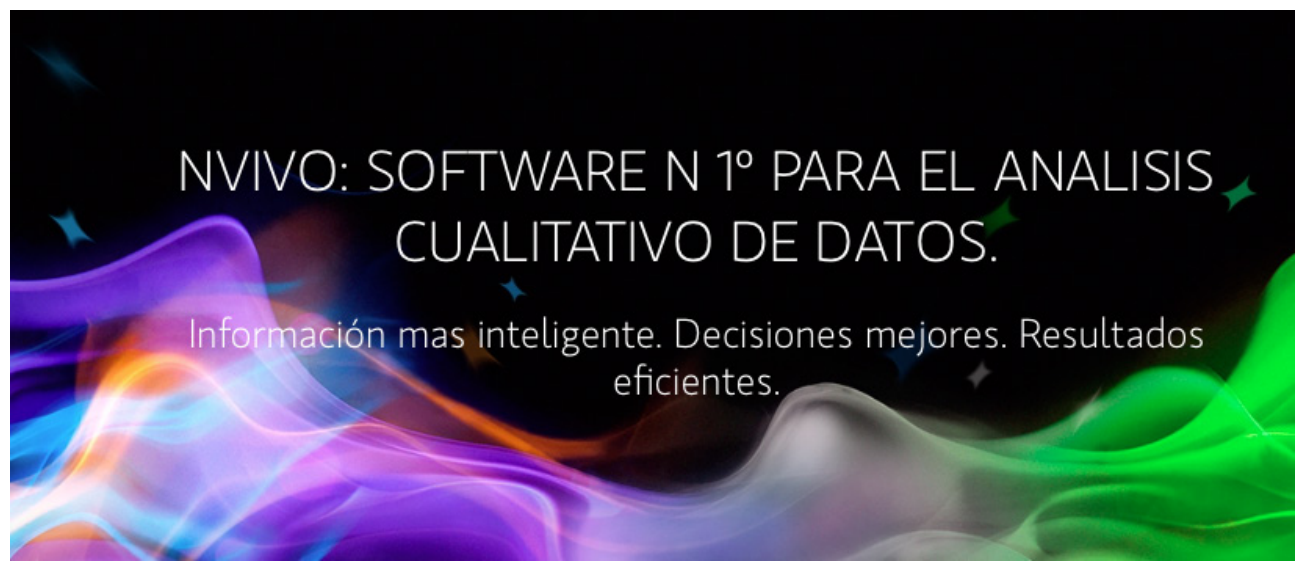

Figura 1. Página de inicio de NVivo.

no solo se obtiene resultados en un menor periodo de tiempo, sino que además nos permite descubrir patrones de respuesta que quizá con una lectura o escucha activa pueda pasarse por alto.

\section{NVivo: El software para la automatización de datos para investigaciones cualitativas}

Gracias a los avances de las tecnologías de la información y la comunicación se vienen desarrollando herramientas capaces de codificar datos electrónicamente dotando a las investigaciones de mayor eficacia y rigor. Uno de estos programas informáticos es NVivo. Se trata de uno de los softwares más utilizados para analizar datos relacionados con investigaciones cualitativas y métodos mixtos (NVivo, 2019, 2019a). Destaca por ser una herramienta capaz de automatizar datos no numéricos como entrevistas, encuestas, y contenido textual y ayudar así a los investigadores (Alyahmady y Saleh, 2013).

Nvivo está desarrollado por QSR International y se puede adquirir desde este enlace (http://www. qsrinternational.com/). Este programa permite, no solo organizar todos los materiales que componen nuestras investigaciones, sino que además nos ayuda a sistematizar, procesar y analizar los datos dándoles forma y sentido (Dias et al. 2016).

Con NVivo podemos importar distintos tipos de datos ya sean estos textuales, audio, imagen, tweets, etc. y evaluarlos en una misma interfaz (Gómez, 2015).

Esto nos permite trabajar desde un mismo panel con una gran cantidad de datos, además, estos datos se pueden convertir fácilmente y de manera automatizada en presentaciones, informes, y/o diagramas (Leech y Onwuegbuzie, 2011).

En esencia, NVivo es una herramienta que ayuda a los investigadores a organizar y manejar sus materiales para que puedan encontrarle un mayor sentido.

NVivo destaca porque mejora en tiempos y en calidad los resultados de las investigaciones que estemos desarrollando con métodos cualitativos. Cuenta con servicios que automatizan los procesos de análisis de los datos obtenidos, permite descubrir patrones, temas y tendencias, por tanto, nos ayuda a resolver las conclusiones de nuestros estudios de manera solvente y eficaz (Walsh 2003).

\begin{tabular}{|c|c|c|}
\hline \multicolumn{3}{|c|}{ Tareas que permite Nvivo para analizar datos } \\
\hline ID & Tarea & Descripción \\
\hline 1 & $\begin{array}{l}\text { Gestión } \\
\text { de datos }\end{array}$ & $\begin{array}{l}\text { Tenemos la posibilidad de ordenary } \\
\text { categorizar los datos para que después } \\
\text { queden agrupados. Se pueden gestionar } \\
\text { entrevistas, encuestas, y todo tipo de } \\
\text { documentos en pdf, word, audio, etc. }\end{array}$ \\
\hline 2 & $\begin{array}{l}\text { Gestión } \\
\text { de ldeas } \\
\text { constantes }\end{array}$ & $\begin{array}{l}\text { Podemos identificar las principales ideas } \\
\text { que se encuentran en los documentos } \\
\text { que subimos a NVivo en base a repeti- } \\
\text { ción de términos. Las ideas surgen como } \\
\text { nubes de etiquetas. A mayor repetición } \\
\text { de una idea mayor tamaño tendrá. }\end{array}$ \\
\hline 3 & $\begin{array}{l}\text { Gráficos } \\
\text { de datos }\end{array}$ & $\begin{array}{l}\text { Existe la opción de codificar los datos. } \\
\text { Una vez se ha llevado a cabo esta acción, } \\
\text { estos datos pueden convertirse en gráficos } \\
\text { y diagramas que nos pueden ayudar a } \\
\text { entender mejor nuestros resultados }\end{array}$ \\
\hline 4 & $\begin{array}{l}\text { Informes } \\
\text { de datos }\end{array}$ & $\begin{array}{l}\text { Los resultados obtenidos del análisis } \\
\text { de nuestros datos pueden transfor- } \\
\text { marse fácilmente en informes finales } \\
\text { con las informaciones más significa- } \\
\text { tivas de nuestra investigación }\end{array}$ \\
\hline 5 & $\begin{array}{l}\text { Trabajo en } \\
\text { equipo }\end{array}$ & $\begin{array}{l}\text { Se pueden desarrollar proyectos conjuntos } \\
\text { entre investigadores, que pueden trabajar } \\
\text { incluso en remoto. Esta opción ayuda } \\
\text { a que todo el análisis desarrollado por } \\
\text { el grupo esté en sintonía y alienado } \\
\text { con los objetivos de todo el equipo }\end{array}$ \\
\hline
\end{tabular}

Como hemos podido constatar existen herramientas 
de análisis de datos cualitativos asistidos por ordenador que ayudan a los investigadores a realizar mejores trabajos de interpretación de resultados. Es por ello que resulta interesante familiarizarse con estos programas informáticos e incorporarlos como una herramienta más de trabajo del investigador. A continuación, pasamos a explicar cómo podemos sacarle el mayor partido posible a NVivo si estamos trabajando con entrevistas semiestructuradas.

\section{Aplicando NVivo a entrevistas semiestructuras}

Utilizar NVivo no es sencillo, es necesario conocer el software y su dinámica. Ante esta circunstancia, presentamos por un lado, un marco de trabajo que sirva de guía para la codificación de entrevista semiestructurada con esta herramienta, y por otro lado un caso práctico que permita contextualizar dicha guía y por tanto hacer más entendible el funcionamiento de la herramienta (Wiltshier, 2011).

NVivo nos ayuda a dar los primeros pasos para categorizar nuestros datos, y sirve para organizar y recuperar material en diferentes formatos. Cada vez que utilizamos NVivo para una investigación ésta se incluye como nuevo proyecto (Sabariego, 2018; Zamawe, 2015).

\begin{tabular}{|c|c|c|}
\hline ID & Tipo de dato & Descripción \\
\hline 1 & Recursos & $\begin{array}{l}\text { Archivo editable de texto } \\
\text { en otro formato }\end{array}$ \\
\hline 2 & Memos & Anotaciones \\
\hline 3 & Nodos o códigos & $\begin{array}{l}\text { Pueden ser temas, tópicos, } \\
\text { conceptos, permite enlazar los } \\
\text { materiales y poder encontrarlos. } \\
\text { Para codificar contamos con } \\
\text { nodos libres (sin organización } \\
\text { previa), nodos de árboles } \\
\text { (categorías o subcategorías), y } \\
\text { Nodos de caso (para diferen- } \\
\text { ciar casos y hacer preguntas) }\end{array}$ \\
\hline
\end{tabular}

$4 \quad$ Atributos

Son las propiedades que tienen
los documentos y los nodos.
Se pueden usar word, pdf,
hojas de cálculo, materiales
de audio y video, páginas
web y artículos en línea.

Las fases para la codificación de las entrevistas semiestructuradas (Gómez, 2015) utilizando NVivo pasan obligatoriamente por los siguientes pasos:

- Agregar entrevistas: La primera fase una vez entramos en la herramienta NVivo pasa por la fase agregar las entrevistas semiestructuradas. Cuando accedemos al Software se despliega la pestaña de proyecto Vacío, y proyecto de prueba (Word y bloc de notas). Para agregar documentos hay que ir a la pestaña de datos y seguidamente a la pestaña documentos. Con ello podremos subir las entrevistas transcritas, después hay que hacer clic. Las entrevistas pueden estar en formato en pdf, en este caos hay que ir a la pestaña datos, hacemos clic en la pestaña pdf, después guardar, y una vez guardado el pdf de la entrevista estaría subida. Por último podemos agregar las entrevistas copiando el texto directamente a un nuevo documento que se genera dentro de la plataforma de NVivo, para ello, una vez estemos conectados a NVivo debemos hacer clic en el botón derecho del ratón, seguidamente hacer clic en elemento interno nuevo, después documento de texto y seguidamente pegamos texto. Es importante recalcar que, si subimos una entrevista en formato bloc de notas o Word sí podremos retocarlo, en cambio si está en pdf no podremos reescribir sobre el documento. te formato no podremos retocarlo.

- Crear categorías y nodos: Una vez hayamos subido todas nuestras entrevistas semiestructuradas tenemos tres opciones para procesar los datos. Primero podemos orientar las categorías, es decir, podemos pedirle a NVivo que por frecuencia de palabras nos reconozca los temas que más se repiten. Para ello debemos ir a la pestaña Consultar que se encuentra en la parte izquierda de la pantalla, después hacemos clic en consulta nueva, y clic en frecuencia de palabras. Una vez hecho esto veremos por frecuencia todos los términos que se van repitiendo de las entrevistas, elegiremos aquellos términos más importantes, al mismo tiempo haremos clic en el botón derecho, y elegiremos la categoría nodo. De esa manera se asociará una palabra a un nodo. Cada vez que subrayemos una frase de las entrevistas y las asociemos a un nodo concreto esa frase quedará categorizar a ese nodo. Al final, las frases importantes de las entrevistas quedarán agrupadas por nodos

- Criterios de frecuencia de palabras/ Árbol de palabras: una vez se agrupen todas las declaraciones importantes de las entrevistas en distintos modos, pasaremos el criterio de frecuencia por cada nodo, y utilizaremos la herramienta Árbol de palabras. Esta herramienta convertirá cada nodo en una frase ramificada muy sintetizada de nuestros datos. La interpretación final de esa frase árbol formará parte de las conclusiones de nuestra investigación.

A continuación, y una vez visto los pasos básicos para la codificación de entrevistas semiestructuradas, vamos a explicar paso a paso cómo implementar nuestros datos con Nvivo. A lo largo de este apartado y de manera sistemática explicaremos dos maneras de enfrentarnos a las entrevistas con este software, por un lado, desde el punto de vista del sistema árbol, y por otro, implementando el servicio de nube de etiquetas. 


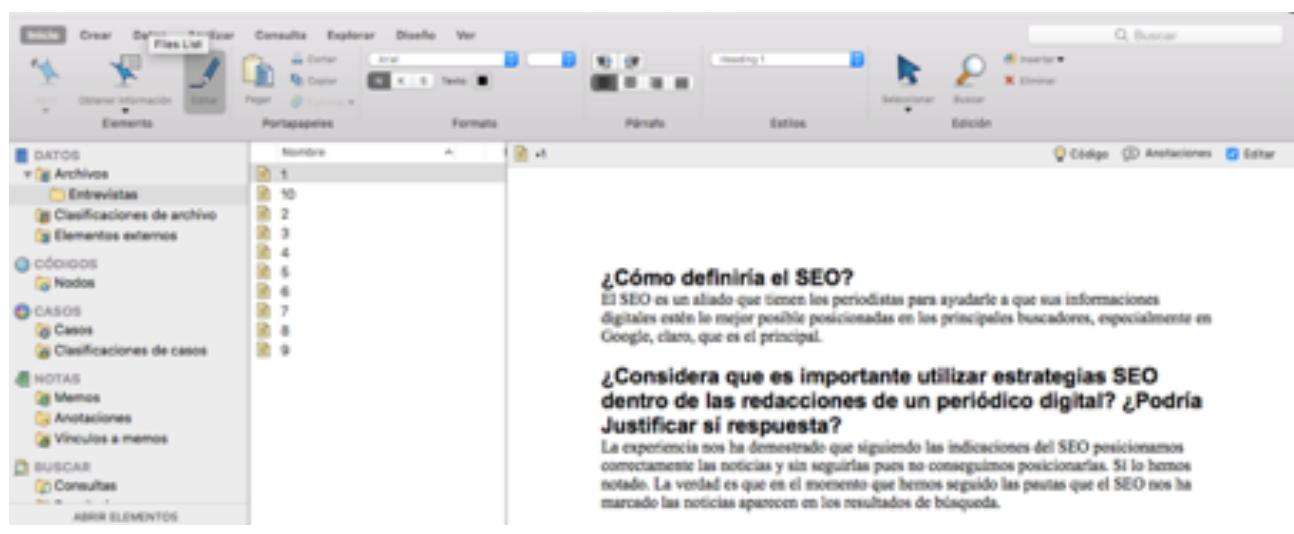

Figura 2. Interfaz de la herramienta Nvivo en la parte central aparecen diez documentos. Cada uno de ellos son una de las entrevistas realizadas y transcritas. En la parte de la derecha se muestra parte de la transcripción de una de las entrevistas.

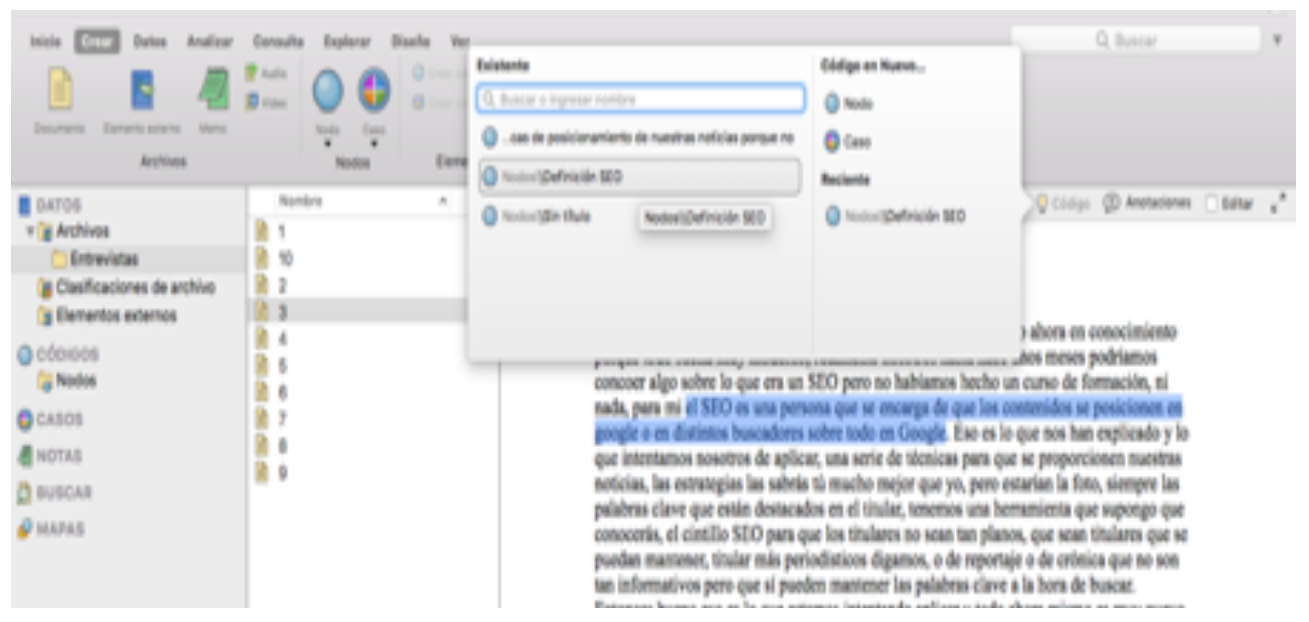

Figura 3. Se muestra la selección de una de las declaraciones más interesantes llevadas a cabo por el entrevistado tres y su consiguiente clasificación temática. En este caso en la clasificación o nodo (descrito así por la herramienta Nvivo) "Definición SEO". Cuando vayamos al Nodo "Definición SEO" aparecerá la declaración subrayada, es decir “...el SEO es una persona que se encarga de que los contenidos se posicionen en Google o distintos buscadores sobre todo en Google."

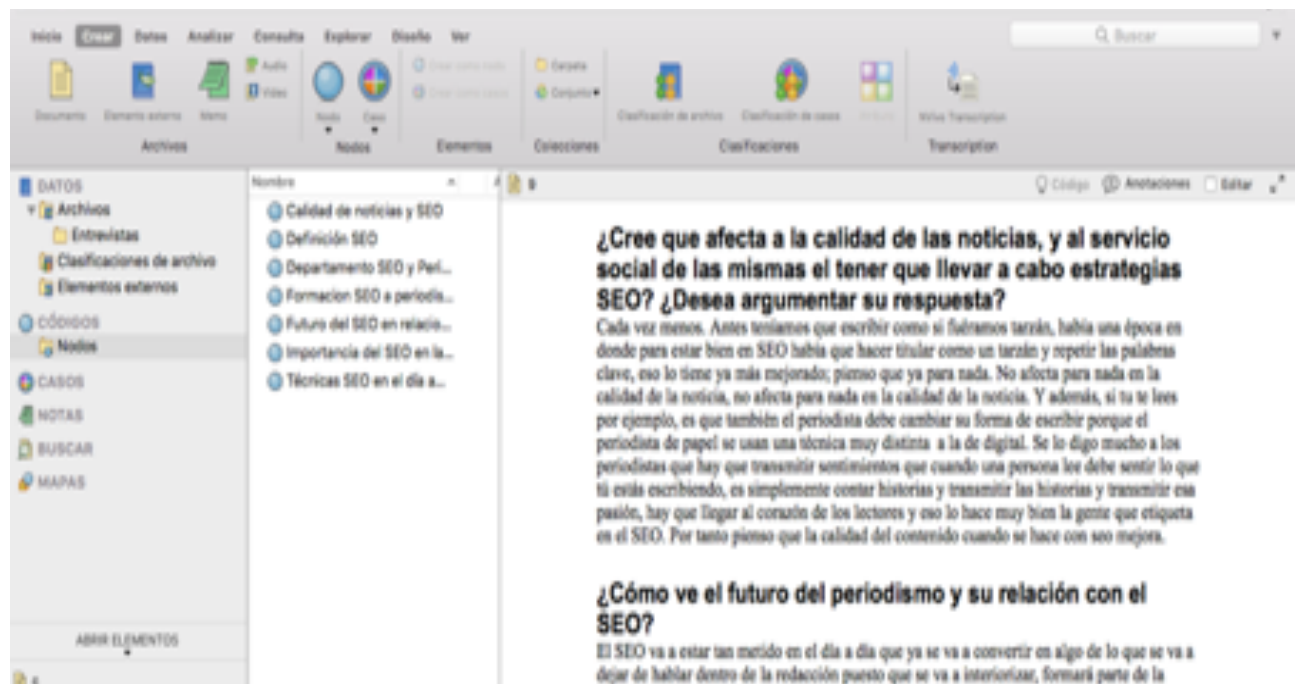

Figura 4. Se muestra todas las categorías creadas e identificadas en base a la información de las entrevistas. 


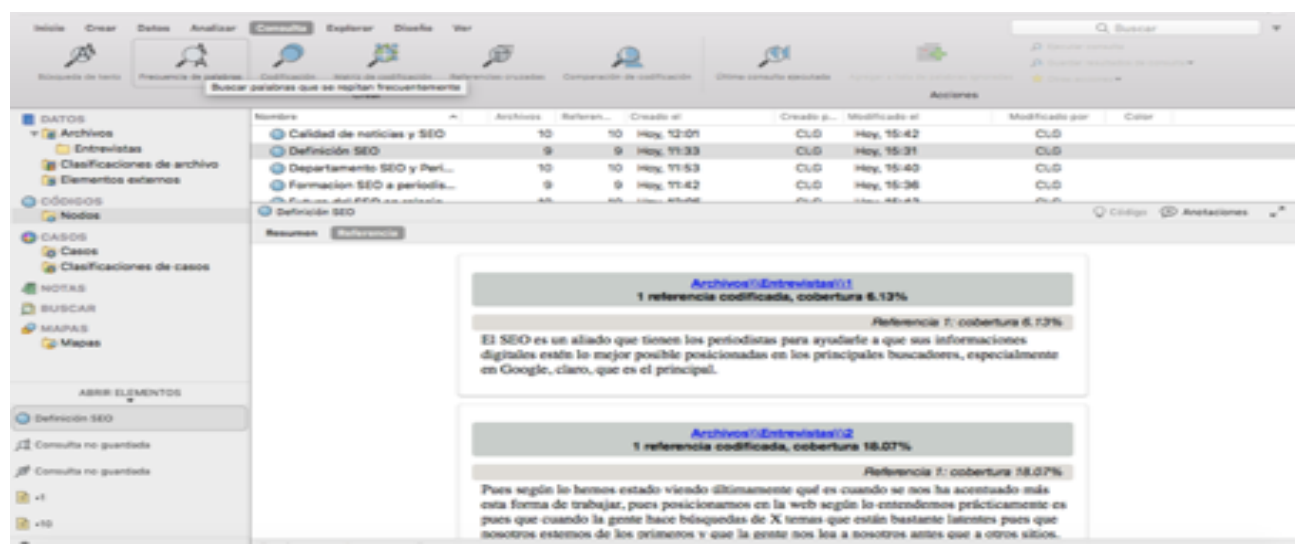

Figura 5. Se muestra el contenido que se ha incluido de cada una de las entrevistas en cada uno de los nodos o categorías descritos. Cada recuadro de referencia codificada recoge declaraciones de cada entrevista.

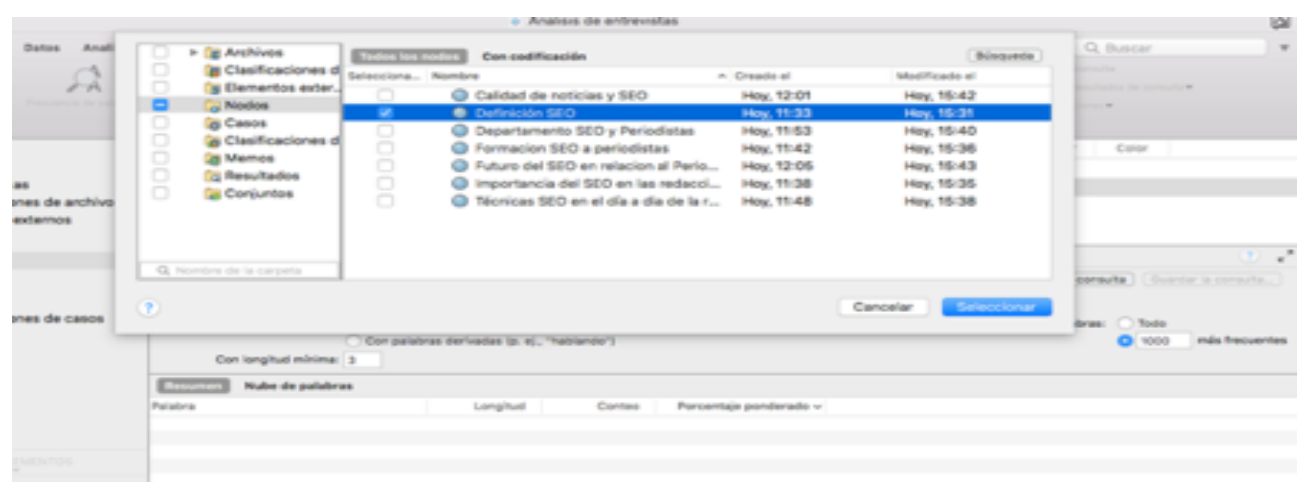

Figura 6. Se muestra la herramienta de conteo de palabras en base a nuestras categorías. En este caso se observa el proceso de conteo de palabras en base a la categoría o nodo "Definición SEO".

Imaginemos que hemos llevado a cabo diez entrevistas a profesionales sobre visibilidad web y cibermedios. Lo primero que tendremos que hacer es volcar todas las entrevistas a la plataforma, ya sea en formato word (Figura 2).

Una vez volcamos todos los textos de las entrevistas en el Software Nvivo, pasamos a identificar las declaraciones más interesantes y a clasificarlas por nodos (Figura 3).

El proceso de selección y categorización temática se lleva a cabo sobre todas las entrevistas. De este modo podemos agrupar todas las declaraciones importantes en distintos temas (Figura 4).

De nuestro análisis de las entrevistas obtenemos en este caso siete categorías temáticas que agrupan las declaraciones de los diez entrevistados (en este caso siete, pero podrían haber sido más o menos según nuestros intereses). En nuestro ejemplo estas categorías o nodos se han dividido en:

- Calidad de noticias y SEO

- Definición SEO

\section{- Departamento SEO y Periodistas}

- Formación SEO a periodistas

- Futuro del SEO en relación al periodismo

- Importancia del SEO en la redacción periodística

- Técnicas SEO en el día a día de la redacción

A continuación, mostramos cómo trabajar los nodos y identificar los resultados. Para ello tomaremos como referencia el nodo "Definición SEO" (Figura 5).

En la imagen se muestran las declaraciones seleccionadas e incluidas en cada categoría, en este caso sobre la definición de SEO. Una vez desplegamos la categoría definición de SEO, nos aparece localizadas todas las declaraciones de los entrevistados que seleccionamos previamente (Figura 6):

En la imagen se muestra el proceso por el cual podemos identificar qué palabras son las más utilizadas por los entrevistados en base a una categoría o nodo. A mayor cantidad de veces repetidas una palabas más posibilidades de que ésta tenga un valor importante (Figura 7): 


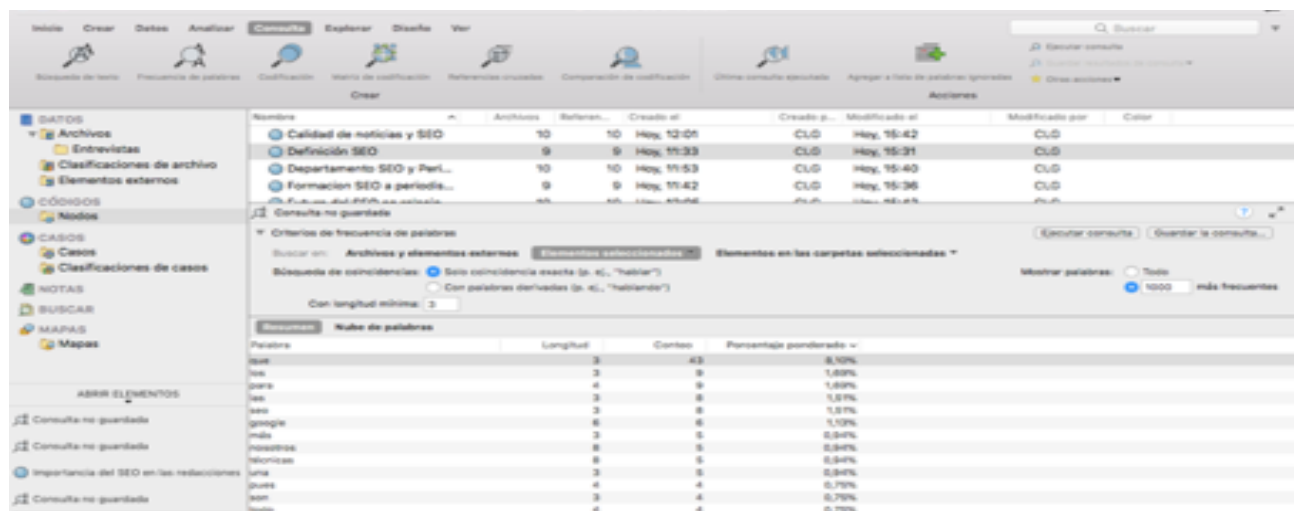

Figura 7. Se muestra el conteo de palabras y su porcentaje de repetición en base al nodo o categoría "Definición SEO".

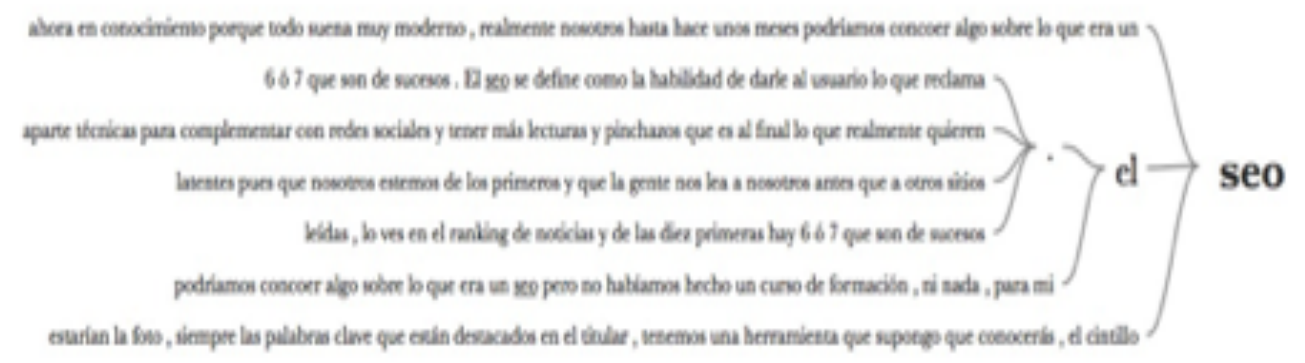

Figura 8. Se muestra el árbol de palabras de la categoría/nodo "definición SEO".

En la imagen se muestra que la palabra SEO tiene una reiteración constante en las declaraciones de los entrevistados. Obviamos las palabras vacías ("que", "los", "para" y "las") ya que no aportan un valor real a las declaraciones. Y seguidamente seleccionamos el término "SEO" y la opción de la herramienta Nvivo "Árbol de palabras" (Figura 8).

En la imagen se muestra que la palabra SEO tiene una reiteración constante en las declaraciones de los entrevistados. Obviamos las palabras vacías ("que", "los", "para" y "las") ya que no aportan un valor real a las declaraciones. Y seguidamente seleccionamos el término "SEO" y la opción de la herramienta Nvivo "Árbol de palabras".

De la codificación y posterior creación del árbol de palabras del nodo "Definición SEO" podemos confirmar que:

- Los entrevistados tienen un buen conocimiento sobre qué es el SEO.

- Los entrevistados consideran el SEO como un conjunto de técnicas para que las noticias aparezcan en los primeros puestos de Google, y que el SEO es la persona que se encarga de que las noticias se posicionen en Google.

Estas pautas ejemplificadas deben aplicarse al resto de nodos, sus correspondientes árboles de palabras ofrecerán conclusiones textuales ramificadas a los investigadores que se harán eco de ellas y podrá incluirlas en las conclusiones de su estudio.

A continuación, pasamos a explicar una segunda modalidad de identificación de temas para entrevistas semiestructuradas en base a frecuencia de términos y obtención de nubes de categorías. Esto nos ayudará a tener una visión completa de qué se ha hablado más durante las entrevistas y el investigador podrá enfocarse a unos temas (los más repetidos) y obviar otros (los menos tratados).

Lo primero que debemos hacer es subir un documento, en este caso, se incluyen las entrevistas en un único documento en pdf que hemos llamado Apuntes sobre SEO. El documento se ha subido a la carpeta de archivos (Figura 9).

Una vez subimos el documento, éste se incorpora a la herramienta y nos aparece dispuesto como se muestra en la imagen siguiente (Figura 10).

Una vez incorporado este manual, llevamos a cabo una consulta de frecuencias de palabras. Esto nos mostrará la frecuencia de uso de palabras dentro del documento interno SEO (Figura 11).

En la imagen se muestra la frecuencia de palabras del documento analizado. En azul se han seleccionado los términos menos relevantes para eliminarlos del listado (Figura 12). 


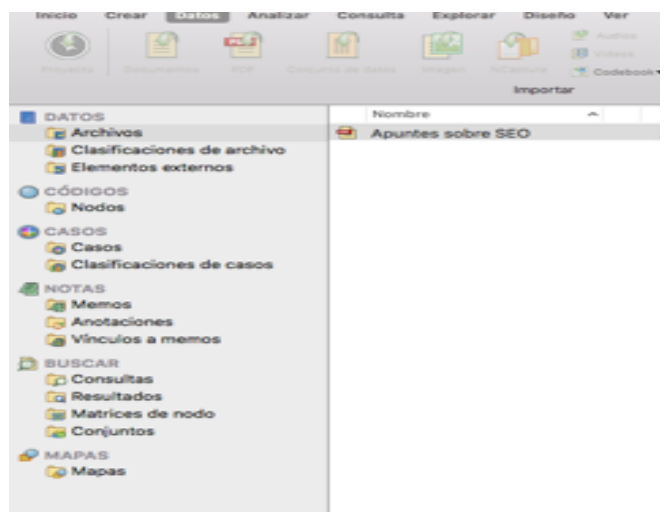

Figura 9. Se muestra la interfaz de NVivo y la manera de subir un archivo pdf.

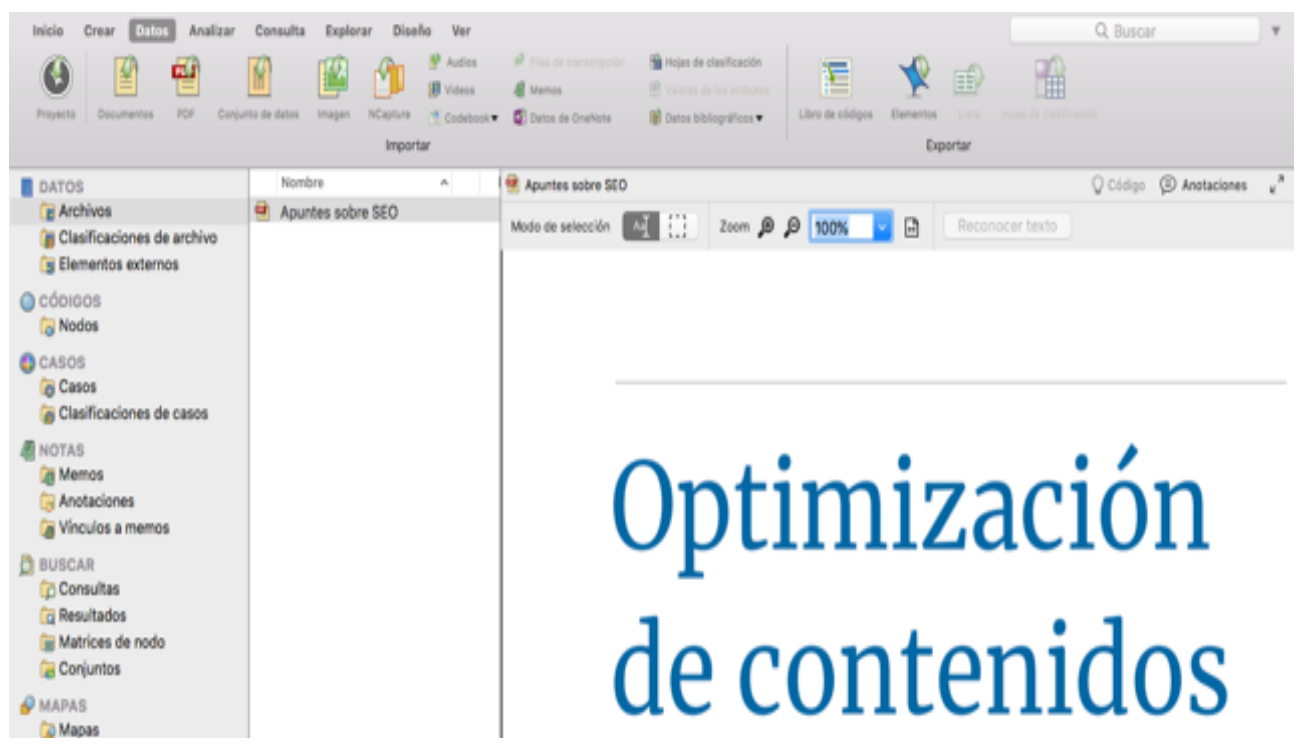

Figura 10. Se muestra identificado el nombre del archivo en pdf y en la parte de la derecha se puede visualizar el contenido del mismo.

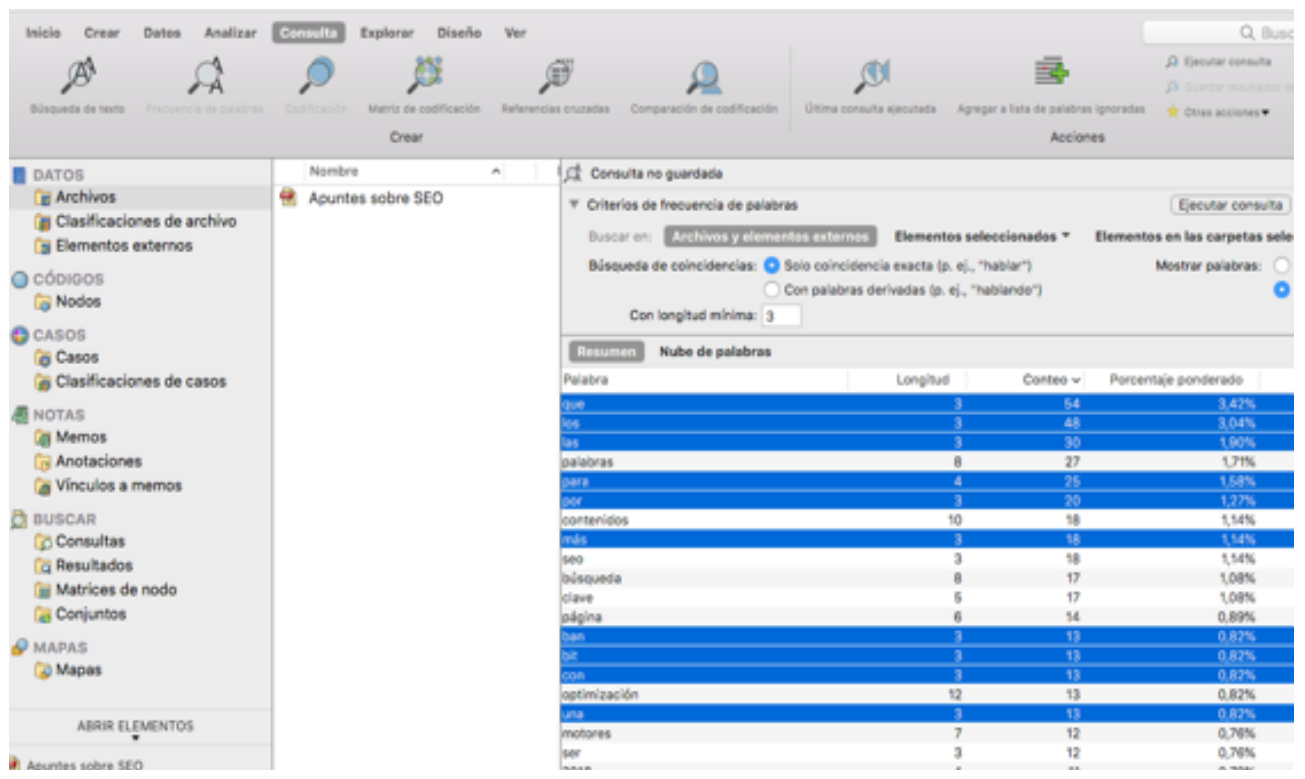

Figura 11. Se muestra el listado de palabras con más frecuencia de uso. En azul se muestran aquellas palabras que no tienen un valor real a nivel conceptual al tratarse de determinantes, preposiciones, etc. es decir palabras vacías. 


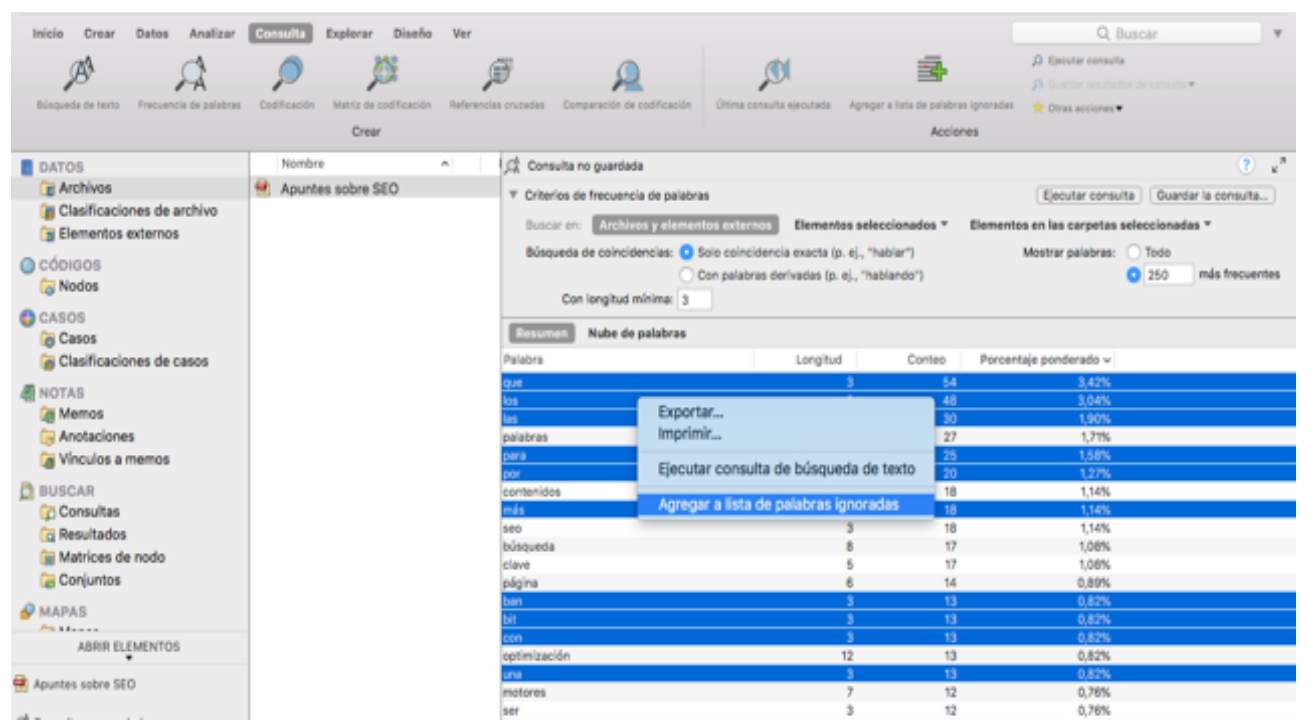

Figura 12. Se muestra la opción de ignorar términos de alta frecuencia poco relevantes, es decir palabras vacías, que se vinculan al documento "Apuntes sobre SEO".

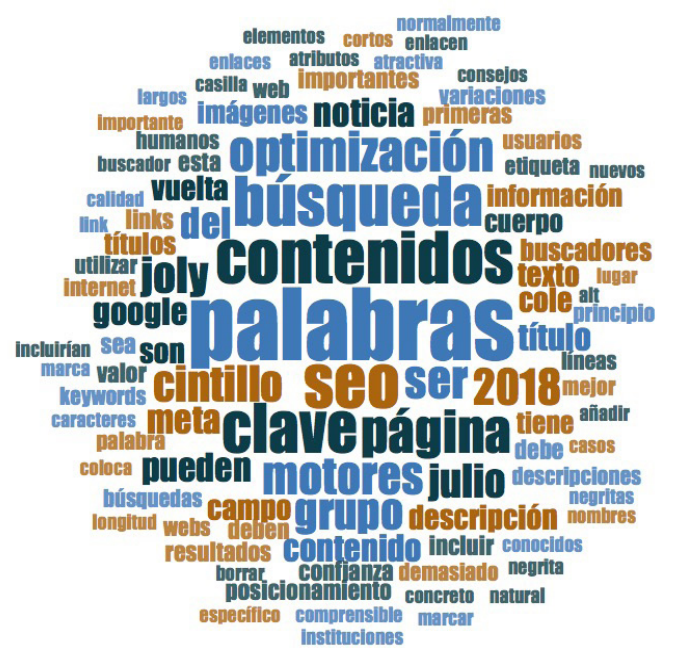

Figura 13. Se muestra la nube de palabras en relación con el documento "Apuntes sobre SEO".

Una vez eliminados los términos de repetición poco relevantes (palabras vacías) creamos una nube de palabras clave que nos indica de manera visual cuáles son los términos más relevantes que recoge el documento analizado (Figura 13).

En la imagen se muestra la nube de palabras que nos permite visualizar las que se usan con más frecuencia. De este modo podemos identificar posibles nodos que puedan tematizar y categorizar las entrevistas de manera eficaz. En este ejemplo por ejemplo sabemos que se ha hablado mucho sobre:

- SEO

- Palabras clave
- Optimización

- Contenidos

- Confianza

- Descripción

- Noticia

- Cintillo

- etc.

Todos estos elementos sirven no solo para entender de qué se ha hablado más durante las entrevistas, sino que además nos puede ayudar y servir de guía para crear los nodos/categorías de las entrevistas. 


\section{Conclusiones}

En este capítulo hemos profundizado en la herramienta de investigación cualitativa NVivo como un aliado para poder codificar entrevistas semiestructuradas y obtener con ello resultados semiautomáticos de las respuestas de los entrevistados. Contamos con la herramienta nube de etiquetas para identificar temas de los que se hablan en las entrevistas y la herramienta árbol de palabra que nos ofrece las respuestas sintetizadas según la categoría/nodo.

Hemos presentado las fases para trabajar de manera eficaz con este software y hemos aplicado dichas fases paso a paso a dos ejemplos prácticos para que los investigadores puedan aplicar in situ este manual básico sobre el uso de NVivo para entrevistas semiestructuradas.

En este sentido, hemos visto por un lado cómo subir las entrevistas transcritas a NVivo, cómo codificarlas en base a categorías/nodos, y cómo obtener las respuestas sistematizadas en forma árbol de palabras. Por otro lado, hemos planteado una estrategia de identificación de posibles nodos/categorías en base a repetición de términos y obtención de nube de categorías por frecuencia de conceptos.

Por último, cabe destacar que NVivo no solo sirve para trabajar con entrevistas semiestructurdas sino que estamos ante una herramienta muy potente capaz de trabajar con muchos otros métodos cualitativos. Y no solo eso, sino que además nos permite codificar muchos otros formatos como son la imagen, el audio, los vídeos, etc. Por lo tanto, es importante recalcar que las posibilidades de uso de este codificador de datos cualitativos tienen una potencialidad muy grande más allá de la investigación con entrevistas.

En definitiva, NVivo se está imponiendo cada vez más como uno de los softwares más interesantes en el mercado para el análisis cualitativo, no solo por su fácil manejo sino también por la cantidad de opciones y servicios con los que cuenta y que van mucho más allá de la codificación y resolución de entrevistas, como por ejemplo informes, mapas conceptuales, visualizaciones y diagramas, entre otros.

\section{Referencias}

AlYahmady H.; Saleh S. (2013). Using NVivo for Data Analysis in Qualitative Research. International Interdisciplinary Journal of Education, 2(2), 181-186.

Dias, J.;Meireles, I.;Ribeiro, M.; Braga, T.; Catafesta, F.;Bernardino, E. (2016) Uso del software NVivo® en una investigación con Teoría Fundamentada. Index de Enermería, $25(4)$

Díaz-Bravo, L.; Torruco-García, U.; Martínez-Hernández,M.; Varela-Ruiz, M. (2003). La entrevista, recurso flexible y dinámico, Investigación en educación médica, 2 (7)

Gómez, O. (2015). Tutorial Nvivo 4 trabajo entrevistas, autocodificación, cruces de información, Disponible en: https:// www.youtube.com/watch?v=2HppQp3zHhM [Fecha de consulta: 16/02/2019].

Leech, N.; Onwuegbuzie, A. (2011). Beyond constant comparison qualitative data analysis: Using NVivo. School Psychology Quarterly, 26 (1), 70-84

NVivo (2019). What is NVivo?, Disponible en: https://www. qsrinternational.com/nvivo/what-is-nvivo [Fecha de consulta: 14/02/2019].

NVivo (2019a). Comience a usar NVivo. Disponible en: https:// www.youtube.com/watch?v=qmWfmkv3NKw [Fecha de consulta: 14/02/2019].

Sabariego, M. (2018) Análisis de datos cualitativos a través del programa NVivo 11 PRO. Dosier 1. Tutorial del programa. Repositorio UB. Disponible en: http://diposit.ub.edu/dspace/ bitstream/2445/118884/1/Dosier\%201.pdf [Fecha de consulta: 15/02/2019].

Walsh, M. (2003). Teaching Qualitative Analysis Using QSR NVivo. The Qualitative Report, V.8 (2), 251-256

Wiltshier, F. (2011). Researching with NVivo. Forum: Qualitative Social Research Sozialforschung, 12(1) http://dx.doi. org/10.17169/fqs-12.1.1628

Zamawe, F. (2015). The Implication of Using NVivo Software in Qualitative Data Analysis: Evidence-Based Reflections. Malawi Medical Journal, 27(1) http://dx.doi.org/10.4314/mmj. v27i1.4 


ISBN 978-84-09-20524-0

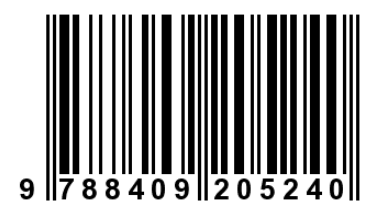

Máster Universitario en Investigación en Comunicación Social (MUCS)

\begin{tabular}{l|ll}
$\mathbf{u} p f$. & $\begin{array}{l}\text { Universitat } \\
\text { Pompeu Fabra } \\
\text { Barcelona }\end{array}$ & $\begin{array}{l}\text { DIGIDOC Grup de Recerca } \\
\text { en Documentació Digital } \\
\text { i Comunicació Interactiva }\end{array}$
\end{tabular} 